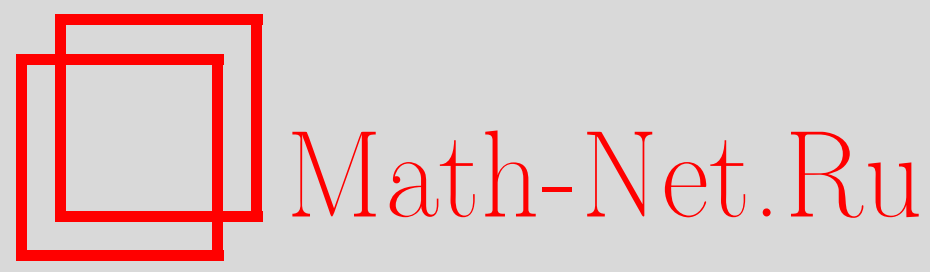

А. И. Герко, Согласованные решения линейных уравнений в вариационных производных, Матем. заметки, 2005, том 77, выпуск 2, 176-187

DOI: https://doi.org/10.4213/mzm2483

Использование Общероссийского математического портала Math-Net.Ru подразумевает, что вы прочитали и согласны с пользовательским соглашением http://www . mathnet.ru/rus/agreement

Параметры загрузки:

IP : 3.80 .181 .102

26 апреля 2023 г., 13:48:48

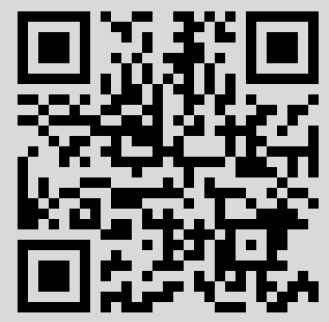


тОм 77 выПУСК 2 фЕВРАль 2005

УДК 517.9

\title{
СОГЛАСОВАННЫЕ РЕШЕНИЯ ЛИНЕЙНЫХ УРАВНЕНИЙ В ВАРИАЦИОННЫХ ПРОИЗВОДНЫХ
}

\section{А. И. Герко}

\begin{abstract}
В заметке для линейных уравнений в вариационных производных рассматривается классическая задача о существовании у уравнения согласованных и равномерно согласованных решений. В частности, мы обобщаем некоторые результаты В. В. Жикова и Б. А. Щербакова о существовании почти периодических, согласованных и равномерно согласованных решений обыкновенных дифференциальных уравнений.

Библиография: 13 названий.
\end{abstract}

1. Введение. Настоящие исследования проведены в рамках разрабатьваемой в Кишиневе программы по изучению многомерных дифференциальных уравнений [1] методами топологической динамики. Основные направления этой программы отражены в обзорной статье [2]. Дополнительно к имеющейся в [2] информации можно добавить вышедшие позже работы [3]-[7]. Во всех перечисленных работах (кроме [7]) рассматриваются многомерные уравнения, у которых независимая переменная ("время") принимает значения из конечномерного векторного пространства. Автором ставится задача качественного исследования систем уравнений в вариационньх производных [8], [9], которые мы записьваем в виде одного уравнения

$$
\frac{\delta y(x)}{\delta x(t)}=f(x, t, y(x)),
$$

где $f: P \times D \times \mathbb{R}^{m} \rightarrow \mathbb{R}^{m}$ - заданное отображение, $y: P \rightarrow \mathbb{R}^{m}$ - неизвестное отображение, $D$ - телесное компактное подмножество из $\mathbb{R}^{n}, D=\overline{\operatorname{int}} D, P$ - пространство непрерьвных отображений $D \rightarrow \mathbb{R}$ с нормой $\sup , \delta y(x) / \delta x(t)$ - вариационная производная отображения $y$. Роль независимой переменной у таких уравнений играет отображениеэлемент бесконечномерного векторного пространства.

Напомним, что вариачионной производной функционала $y: P \rightarrow \mathbb{R}$ в точке $x_{0}$ называется функционал $g: D \rightarrow \mathbb{R}$, удовлетворяющий равенству

$$
\delta y\left(x_{0} ; h\right)=\int_{D} g(t) h(t) d t, \quad h \in P,
$$

где $\delta y\left(x_{0} ; h\right)$ - вариация функционала $y$ в точке $x_{0}$ в направлении $h \in P$, т.е.

$$
\delta y\left(x_{0} ; h\right)=\lim _{\tau \rightarrow 0} \tau^{-1}\left(y\left(x_{0}+\tau h\right)-y\left(x_{0}\right)\right), \quad \tau \in \mathbb{R} .
$$


Некоторые вопросы общей теории линейных уравнений в вариационньх производных изложены в [8] и [9]. Там, а также в [1], имеется и библиография по уравнениям в вариационных производных и их приложениям. Отметим лишш, что уравнения в вариационных производных широко используются в различных разделах теоретической физики.

В статье разрабатывается задача о существовании у уравнения согласованных и равномерно согласованных решений [10] - классическая задача о существовании у уравнений решений, обладающих свойствами возвращаемости, которьми обладает "правая часть" уравнения. В частности, мы на линейные уравнения в вариационных производных распространяем соответствующие результаты В. В. Жикова и Б. А. Щербакова для обыкновенных дифференциальных уравнений из [11, теорема 3, гл. 8$]$ и [10, теоремы 4.3.2-4.3.4].

2. Основные соглашения и вспомогательные утверждения. Известно [9], [8], что уравнение $(1)$ в случае непрерьвной функции $f$ равносильно уравнению в полных производных

$$
y^{\prime} h=F(x, y) h
$$

где

$$
F(x, y) h=\int_{D} f(x, t, y(x)) h(t) d t, \quad h \in P .
$$

В дальнейшем считаем, что правые части $f$ уравнений вида (1) принадлежат пространству $C_{c}\left(P \times D \times \mathbb{R}^{m}, \mathbb{R}^{m}\right)$ непрерьвных отображений $P \times D \times \mathbb{R}^{m} \rightarrow \mathbb{R}^{m}$, снабженному равномерностью компактной сходимости (компактно-открытой топологией). Считаем, что решения уравнения (1) принадлежат пространству $C_{c}\left(P, \mathbb{R}^{m}\right)$ непрерывньх отображений $P \rightarrow \mathbb{R}^{m}$, снабженному равномерностью компактной сходимости. Правые части $F$ уравнений вида $(2)$ считаем принадлежащими пространству $C_{c}\left(P \times D, L_{b}(P\right.$, $\left.\mathbb{R}^{m}\right)$ ) непрерывных отображений $P \times D \rightarrow L_{b}\left(P, \mathbb{R}^{m}\right)$, снабженному равномерностью компактной сходимости. Здесь $L_{b}\left(P, \mathbb{R}^{m}\right)$ - пространство непрерывных линейных отображений $P \rightarrow \mathbb{R}^{m}$, снабженное равномерностью ограниченной сходимости. Естественно, решения уравнения (2) принадлежат $C_{c}\left(P, \mathbb{R}^{m}\right)$.

В дальнейшем $S$ - подполугруппа адлитивной группы $P, 0 \in S$. Каждому отображению $f$ из рассматриваемьх нами функциональных пространств и каждому $s \in S$ можно сопоставить с помощью так назьваемого отображения сдвига $\sigma$ по аргументу из $P$ некоторое отображение $f_{s}$, которое определяется следующим образом. Если $f: P \rightarrow Y$, то $f_{s}(p)=f(s+p), p \in P$. Если $f: P \times D \rightarrow Y$, то $f_{s}(p, t)=f(s+p, t), p \in P, t \in D$. Через $f S$ обозначаем множество $\left\{f_{s} \mid s \in S\right\}$ и соответственно $(f, \varphi) S=\left\{\left(f_{s}, \varphi_{s}\right) \mid s \in S\right\}$.

Основные результаты мы получим для линейных уравнений в вариационных производных

$$
\frac{\delta y(x)}{\delta x(t)}=a(x, t) y(x)+b(x, t),
$$

где $a \in C_{c}\left(P \times D, L_{b}\left(\mathbb{R}^{m}, \mathbb{R}^{m}\right)\right), b \in C_{c}\left(P \times D, \mathbb{R}^{m}\right)$.

Наряду с уравнением $(3)$ для любых $(c, d) \in \overline{(a, b) S}$ мы рассматриваем также уравнения

$$
\begin{aligned}
& \frac{\delta y(x)}{\delta x(t)}=c(x, t) y(x), \\
& \frac{\delta y(x)}{\delta x(t)}=c(x, t) y(x)+d(x, t)
\end{aligned}
$$


(здесь $\left.(a, b) S=\left\{\left(a_{s}, b_{s}\right) \mid s \in S\right\}\right)$.

Нам понадобится понятие согласованности решения уравнения с "правой частью" уравнения. Обычно [10], [3] при определении понятия согласованности используются динамические системы. Наше определение не связано с динамическими системами.

ОПРЕДЕЛЕНИЕ 1. Пусть $\varphi \in C_{c}\left(P, \mathbb{R}^{m}\right)$ и $(a, b) \in C_{c}\left(P \times D, L_{b}\left(\mathbb{R}^{m}, \mathbb{R}^{m}\right) \times \mathbb{R}^{m}\right)$. Говорим, что отображение $\varphi S$-согласовано с $(a, b)$, если для произвольного окружения $\alpha$ равномерной структуры пространства $C_{c}\left(P, \mathbb{R}^{m}\right)$ существует окружение $\beta$ равномерной структуры пространства $C_{c}\left(P \times D, L_{b}\left(\mathbb{R}^{m}, \mathbb{R}^{m}\right) \times \mathbb{R}^{m}\right)$ такое, что из $s \in S$ и $\left((a, b),\left(a_{s}, b_{s}\right)\right) \in \beta$ следует соотношение $\left(\varphi, \varphi_{s}\right) \in \alpha$. Говорим, что отображение $\varphi$ равномерно $S$-согласовано с $(a, b)$, если для произвольного окружения $\alpha$ равномерной структуры пространства $C_{c}\left(P, \mathbb{R}^{m}\right)$ существует окружение $\beta$ равномерной структуры пространства $C_{c}\left(P \times D, L_{b}\left(\mathbb{R}^{m}, \mathbb{R}^{m}\right) \times \mathbb{R}^{m}\right)$ такое, что из $s, t \in S$ и $\left(\left(a_{t}, b_{t}\right),\left(a_{s}, b_{s}\right)\right) \in \beta$ следует соотношение $\left(\varphi_{t}, \varphi_{s}\right) \in \alpha$.

Понятно, что отображение $\varphi S$-согласовано с $(a, b)$ тогда и только тогда, когда для произвольных $\varepsilon>0$ и компакта $A \subset P$ существуют число $\delta>0$, компакт $M \times Q \subset P \times D$ и ограниченное множество $T \subset \mathbb{R}^{m}$ такие, что для любого $s \in S$, для которого

$$
\left\|a(m, q) h-a_{s}(m, q) h\right\|<\delta \quad \text { и } \quad\left\|b(m, q)-b_{s}(m, q)\right\|<\delta
$$

при всех $(m, q, h) \in M \times Q \times T$, вьполняется для всех $p \in A$ соотношение

$$
\left\|\varphi(p)-\varphi_{s}(p)\right\|<\varepsilon .
$$

Отображение $\varphi$ равномерно $S$-согласовано с $(a, b)$ тогда и только тогда, когда для произвольных $\varepsilon>0$ и компакта $A \subset P$ сушествуют число $\delta>0$, компакт $M \times Q \subset P \times D$ и ограниченное множество $T \subset \mathbb{R}^{m}$ такие, что для любых $s, t \in S$, для которых

$$
\left\|a_{t}(m, q) h-a_{s}(m, q) h\right\|<\delta \quad \text { и } \quad\left\|b_{t}(m, q)-b_{s}(m, q)\right\|<\delta
$$

при всех $(m, q, h) \in M \times Q \times T$, вьполняется для всех $p \in A$ соотношение

$$
\left\|\varphi_{t}(p)-\varphi_{s}(p)\right\|<\varepsilon .
$$

Суть понятия $S$-согласованности состоит в том, что если $\varphi S$-согласовано (равномерно $S$-согласовано) с $f$ и $f$ обладает определенным свойством возвращаемости, то и $\varphi$ обладает этим свойством возвращаемости. Поясним сказанное более подробно.

Пусть $X$ и $Y$ - некоторые пространства отображений, на которых определено действие полугрупшы $S$ с помощью отображения сдвига $\sigma, \mathcal{U}[X]$ и $\mathscr{U}[Y]-$ равномерные структуры пространств $X$ и $Y$ соответственно, $[S]$ - некоторьй класс подмножеств из $S$, $f \in X$ (либо $f \in Y$ ).

Отображение $f$ назьваем $[S]$-рекурсивным, если для произвольного окружения $\alpha \in$ $\mathscr{U}[X]$ существует множество $A \in[S]$, для которого $\left(f, f_{a}\right) \in \alpha$ при всех $a \in A$. Множество $f S$ назьваем $[S]$-рекурсивным, если для произвольного окружения $\alpha \in \mathscr{U}[X]$ существует множество $A \in[S]$, для которого $\left(f_{s}, f_{s+a}\right) \in \alpha$ при всех $s \in S$ и $a \in A$.

И пусть $\varphi \in X, f \in Y$. Тогда

1) если $\varphi S$-согласовано с $f$ и отображение $f[S]$-рекурсивно, то отображение $\varphi$ $[S]$-рекурсивно;

2 ) если $\varphi$ равномерно $S$-согласовано с $f$ и множество $f S[S]$-рекурсивно, то множество $\varphi S[S]$-рекурсивно. 
Последние определения и утверждение хорошо согласуются с известными понятиями и фактами для динамических систем [12].

В качестве конкретизаций $[S]$-рекурсивности могут выступать различные типы устойчивости по Пуассону отображений, в частности, $S Q$-устойчивость по Пуассону, $S$-устойчивость по Пуассону, $S Q$-рекуррентность в смысле Биркгофа, $S Q$-почти периодичность в смысле Бора (здесь $Q$ - подмножество из $S$ ) (подробнее см. [12], [13]).

Таким образом, если установлено, что некоторое решение $\varphi$ уравнения $S$-согласовано (равномерно $S$-согласовано) с "правой частью" $f$ этого уравнения и отображение $f$ $S Q$-устойчиво по Пуассону, либо $S$-устойчиво по Пуассону, либо $S Q$-рекуррентно в смысле Биркгофа ( $S Q$-почти периодично в смысле Бора), то и решение $\varphi$ соответственно $S Q$-устойчиво по Пуассону, либо $S$-устойчиво по Пуассону, либо $S Q$-рекуррентно в смысле Биркгофа (соответственно $S Q$-почти периодично в смысле Бора).

ОПРЕДЕЛЕНИЕ 2. ФУнкция $\varphi \in C_{c}\left(P, \mathbb{R}^{m}\right)$ называется ограниченной, если ограничено множество $\overline{\varphi(P)}$. Функция $\varphi \in C_{c}\left(P, \mathbb{R}^{m}\right)$ (соответственно $a \in C_{c}\left(P \times D, L_{b}\left(\mathbb{R}^{m}\right.\right.$, $\left.\left.\left.\mathbb{R}^{m}\right)\right),(a, b) \in C_{c}\left(P \times D, L_{b}\left(\mathbb{R}^{m}, \mathbb{R}^{m}\right) \times \mathbb{R}^{m}\right)\right)$ назьвается $S$-устойчивой по Лагранжу если компактно множество $\overline{\varphi S}$ (соответственно $\overline{a S}, \overline{(a, b) S}$ ).

\section{Имеет место следующая}

Лемма 1. Если отображсение ч равномерно $S$-согласовано с $S$-устойчивым по Лагранжу отображснием $(a, b)$, то оно $S$-устойчиво по Лагранжу.

ДокАЗАТЕЛЬСтво. Пусть $\alpha$ - произвольное окружение равномерной структуры пространства $C_{c}\left(P, \mathbb{R}^{m}\right)$ и $\beta$ - соответствующее ему в силу равномерной $S$-согласованности $\varphi$ с $(a, b)$ окружение равномерной структуры пространства $C_{c}\left(P \times D, L_{b}\left(\mathbb{R}^{m}\right.\right.$, $\left.\left.\left.\mathbb{R}^{m}\right) \times \mathbb{R}^{m}\right)\right)$. И пусть $\left\{\varphi_{s_{n}}\right\}$ - произвольная направленность в $\varphi S$. Так как множество $(\overline{(a, b) S})$ компактно, то из направленности $\left\{\left(a_{s_{n}}, b_{s_{n}}\right)\right\}$ можно выделить сходящуюся поднаправленность $\left\{\left(a_{s_{n_{k}}}, b_{s_{n_{k}}}\right)\right\}$. Тогда для окружения $\beta$ найдется $k_{0}$ такое, что для произвольных $k>k_{0}$ и $m>k_{0}$ выполняется соотношение

$$
\left(\left(a_{s_{n_{k}}}, b_{s_{n_{k}}}\right),\left(a_{s_{n_{m}}}, b_{s_{n_{m}}}\right)\right) \in \beta \text {. }
$$

В таком случае $\left(\varphi_{s_{n_{k}}}, \varphi_{s_{n_{m}}}\right) \in \alpha$ для произвольных $k>k_{0}$ и $m>k_{0}$. Это означает, что направленность $\left\{\varphi_{s_{n_{k}}}\right\}$ является направленностью Коши. Итак, из произвольной направленности в $\varphi S$ можно выделить поднаправленность Коши. Поэтому множество $\varphi S$, а следовательно, и $\overline{\varphi S}$ вполне ограничено. Но тогда компактность $\overline{\varphi S}$ следует из его полноты.

3. Результаты. Важную роль в наших доказательствах будет играть следующая лемма замкнутости, которая вытекает из леммы 2 из [7] и сделанного вьше замечания о эквивалентности уравнения типа (1) уравнению в полных производных типа (2).

Лемма 2. Пусть $I$ - направленное множество, $K \subset \mathbb{R}^{m}$ - компактное множсество и для всех $i \in I$ отобрахсение $\varphi_{i}: P \rightarrow K$ - решение уравнения

$$
\frac{\delta y(x)}{\delta x(t)}=f_{i}(x, t, y(x)) .
$$

Eсли $\lim _{i} f_{i}=f$ в $C_{c}\left(P \times D \times \mathbb{R}^{m}, \mathbb{R}^{m}\right), m o$

1) семейство $\overline{\left\{\varphi_{i} \mid i \in I\right\}}$ компактно; 
2) предел $\varphi$ произвольной поднаправленности направленности $\left\{\varphi_{i}\right\}$ является решением уравнения

$$
\frac{\delta y(x)}{\delta x(t)}=f(x, t, y(x))
$$

ТЕОРемА 1. Пусть для уравнения (3), имеющего ограниченное решение $\varphi$, выполнены условия

1) отобрахсние а S-устойчиво по Лагранжу;

2) для всех $c \in \overline{a S}$ уравнение (4) не имеет ненулевых ограниченных решений.

Тогда для всех $(c, d) \in \overline{(a, b) S}$ уравнение (5) имеет единственное ограниченное решение. Это решение равномерно $S$-согласовано $c(c, d)$ и является $S$-устойчивым по Лагранжу, если отображение $(a, b)$ S-устойчиво по Лагранжу.

ДокАЗАТЕЛЬСТво. Из условия теоремы следует, что $\varphi$ - единственное ограниченное решение уравнения (3). Обозначим

$$
k_{0}=\sup _{\psi \in \overline{\varphi S}}\|\psi\|
$$

Предположим, что $\varphi$ не равномерно $S$-согласовано с $(a, b)$. Тогда существуют число $\varepsilon>0$ и компакт $A \subset P$ такие, что для произвольных натурального числа $n \in \mathbb{N}$, компакта $M \times Q \subset P \times D$ и ограниченного множества $T \subset \mathbb{R}^{m}$ существуют элементы $s_{n}, t_{n} \in S$ такие, что для всех $(m, q, h) \in M \times Q \times T$

$$
\left\|a_{t_{n}}(m, q) h-a_{s_{n}}(m, q) h\right\|<\frac{1}{n}, \quad\left\|b_{t_{n}}(m, q)-b_{s_{n}}(m, q)\right\|<\frac{1}{n}
$$

и для некоторого $p \in A$

$$
\left\|\varphi_{t_{n}}(p)-\varphi_{s_{n}}(p)\right\| \geqslant \varepsilon .
$$

Рассмотрим для произвольного $n \in \mathbb{N}$ отображение $b_{n}: P \times D \rightarrow \mathbb{R}^{m}$, определяемое правилом

$$
b_{n}(x, t)=\left(a_{t_{n}}(x, t)-a_{s_{n}}(x, t)\right) \varphi_{s_{n}}(x)+b_{t_{n}}(x, t)-b_{s_{n}}(x, t),
$$

и докажем, что $\lim _{n \rightarrow \infty} b_{n}$ в $C_{c}\left(P \times D, \mathbb{R}^{m}\right)$ равен 0 , т.е. нулевому отображению.

Пусть $\delta>0$ - произвольное число, $T=\left\{x \mid x \in \mathbb{R}^{m} \wedge\|x\| \leqslant 1\right\}$ и натуральное число $n_{0}$ таково, что $\left(k_{0}+1\right) / n_{0}<\delta$. Тогда для всех $(m, q) \in M \times Q$ и произвольного $n>n_{0}$ с учетом соотношений $(8)$ получим

$$
\begin{aligned}
\left\|b_{n}(m, q)\right\| & =\left\|\left(a_{t_{n}}(m, q)-a_{s_{n}}(m, q)\right) \varphi_{s_{n}}(x)+b_{t_{n}}(m, q)-b_{s_{n}}(m, q)\right\| \\
& \leqslant\left\|a_{t_{n}}(m, q)-a_{s_{n}}(m, q)\right\|\left\|\varphi_{s_{n}}(x)\right\|+\left\|b_{t_{n}}(m, q)-b_{s_{n}}(m, q)\right\| \\
& <\sup _{h \in T}\left\|\left(a_{t_{n}}(m, q)-a_{s_{n}}(m, q)\right) h\right\| \cdot k_{0}+\frac{1}{n} \leqslant \frac{k_{0}}{n}+\frac{1}{n}<\delta,
\end{aligned}
$$

т.е. $\left\|b_{n}(m, q)\right\|<\delta$. Доказанное и означает, что $\lim _{n \rightarrow \infty} b_{n}=0$.

Поскольку отображение $a S$-устойчиво по Лагранжу, то, не нарушая общности рассуждений, можем считать, что

$$
\lim _{n \rightarrow \infty} a_{t_{n}}=c \in \overline{a S}
$$


Поэтому

$$
\lim _{n \rightarrow \infty}\left(a_{t_{n}}, b_{n}\right)=(c, 0) .
$$

Рассмотрим для всех $n \in \mathbb{N}$ уравнение

$$
\frac{\delta y(x)}{\delta x(t)}=a_{t_{n}}(x, t) y(x)+b_{n}(x, t)
$$

и докажем, что отображение $\varphi_{n}=\varphi_{t_{n}}-\varphi_{s_{n}}$ является его решением.

Заметим, что

1) уравнение (3) равносильно уравнению в полных производных

$$
y^{\prime} h=A(x) h y+B(x) h
$$

2) уравнение

$$
\frac{\delta y(x)}{\delta x(t)}=a_{t_{n}}(x, t) y(x)+b_{t_{n}}(x, t)
$$

равносильно уравнению в полных производных

$$
y^{\prime} h=A_{t_{n}}(x) h y+B_{t_{n}}(x) h
$$

3) уравнение

$$
\frac{\delta y(x)}{\delta x(t)}=a_{s_{n}}(x, t) y(x)+b_{s_{n}}(x, t)
$$

равносильно уравнению в полных производных

$$
y^{\prime} h=A_{s_{n}}(x) h y+B_{s_{n}}(x) h
$$

4) уравнение (11) равносильно уравнению в полных производных

$$
y^{\prime} h=A_{t_{n}}(x) h y+\left(A_{t_{n}}(x)-A_{s_{n}}(x)\right) h \varphi_{s_{n}}(x)+\left(B_{t_{n}}(x)-B_{s_{n}}(x)\right) h,
$$

где для всех $h \in P$

$$
\begin{aligned}
A(x) h & =\int_{D} a(x, t) h(t) d t, & B(x) h & =\int_{D} b(x, t) h(t), d t, \\
A_{t_{n}}(x) h & =\int_{D} a_{t_{n}}(x, t) h(t) d t, & B_{t_{n}}(x) h & =\int_{D} b_{t_{n}}(x, t) h(t) d t, \\
A_{s_{n}}(x) h & =\int_{D} a_{s_{n}}(x, t) h(t) d t, & B_{s_{n}}(x) h & =\int_{D} b_{s_{n}}(x, t) h(t) d t .
\end{aligned}
$$


Если мы докажем, что $\varphi_{n}-$ решение уравнения (14), то тем самым будет доказано, что $\varphi_{n}$ является решением уравнения (11).

Учитывая, что $\varphi_{t_{n}}$ и $\varphi_{s_{n}}$ - решения уравнений (12) и (13) соответственно, получим

$$
\begin{aligned}
\varphi_{n}^{\prime}(x) h= & \left(\varphi_{t_{n}}(x)-\varphi_{s_{n}}(x)\right)^{\prime} h=\varphi_{t_{n}}^{\prime}(x) h-\varphi_{s_{n}}^{\prime}(x) h \\
= & A_{t_{n}}(x) h \varphi_{t_{n}}(x)+B_{t_{n}}(x) h-A_{s_{n}}(x) h \varphi_{s_{n}}(x)-B_{s_{n}}(x) h \\
= & A_{t_{n}}(x) h \varphi_{t_{n}}(x)-A_{t_{n}}(x) h \varphi_{s_{n}}(x)+A_{t_{n}}(x) h \varphi_{s_{n}}(x) \\
& -A_{s_{n}}(x) h \varphi_{s_{n}}(x)+\left(B_{t_{n}}(x)-B_{s_{n}}(x)\right) h \\
= & A_{t_{n}}(x) h\left(\varphi_{t_{n}}(x)-\varphi_{s_{n}}(x)\right)+\left(A_{t_{n}}(x)-A_{s_{n}}(x)\right) h \varphi_{s_{n}}(x) \\
& +\left(B_{t_{n}}(x)-B_{s_{n}}(x)\right) h
\end{aligned}
$$

значит, $\varphi_{n}$ - решение уравнения (14) и, следовательно, уравнения (11).

В силу соотношения (10) и леммы 2 из последовательности $\left\{\varphi_{n}\right\}$ можно выделить подпоследовательность $\left\{\varphi_{n_{i}}\right\}$, сходящуюся к некоторому ограниченному решению $\psi$ уравнения (4). Так как уравнение (4) имеет только нулевое ограниченное решение, то $\psi=0 ;$ следовательно,

$$
\lim _{i \rightarrow \infty} \varphi_{n_{i}}=0 .
$$

В таком случае для $\varepsilon$ и компакта $A$ найдется $i_{0} \in \mathbb{N}$ такое, что для произвольных $i>i_{0}$ и $p \in A$ вьполняется соотношение

$$
\left\|\varphi_{n_{i}}(p)\right\|=\left\|\varphi_{t_{n_{i}}}(p)-\varphi_{s_{n_{i}}}(p)\right\|<\varepsilon
$$

противоречащее соотношению (9). Полученное противоречие говорит о том, что $\varphi$ равномерно $S$-согласовано с $(a, b)$.

Если отображение $(a, b) S$-устойчиво по Лагранжу, то по лемме 1 решение $\varphi S$-устойчиво по Лагранжу.

Пусть $(c, d) \in \overline{(a, b) S}$. Тогда в силу леммы 2 уравнение $(5)$ имеет в множестве $\overline{\varphi S}$ ограниченное решение. Кроме этого отображение $c S$-устойчиво по Лагранжу. Значит, для уравнения (5) выполнены те же условия, что и для уравнения (3). Поэтому из доказанного вьше и следует заключение нашей теоремы.

ОПРЕДЕЛЕНИЕ 3. Функция $\varphi \in C_{c}\left(P, \mathbb{R}^{m}\right)$ назьвается $S$-отделенной от нуля, если

$$
\inf _{s \in S}\|\varphi(s)\|>0
$$

ТЕОРема 2. Пусть для уравнения (3), имеющего ограниченное решение $\varphi$, выполнены условия

1) отображсние а S-устойчиво по Лагранжу и для всех $c \in \overline{a S}$ выполнено $\overline{c S}=\overline{a S}$

2) при с = а уравнение (4) не имеет ненулевых ограниченных решений;

3) для всех $c \in \overline{a S}$ каждое ненулевое ограниченное решение уравнения (4) S-отделено от нуля.

Тогда для всех $(c, d) \in \overline{(a, b) S}$ уравнение $(5)$ имеет единственное ограниченное решение. Это решение равномерно $S$-согласовано $c(c, d)$ и является $S$-устойчивым по Лагранжу, если отображение $(a, b)$ S-устойчиво по Лагранжу. 
ДокАЗАтЕльСтво. Пусть $\psi \neq 0$ - ограниченное решение уравнения (4) при $c \in \overline{a S}$. Тогда в силу равенства $\overline{c S}=\overline{a S}$ выполнено

$$
a=\lim _{n} c_{s_{n}}
$$

для некоторой направленности $\left\{s_{n}\right\}$ в $S$. Согласно лемме 2 из направленности $\left\{\psi_{s_{n}}\right\}$ можно выделить поднаправленность $\left\{\psi_{s_{n_{i}}}\right\}$, сходящуюся к некоторому решению $\eta$ уравнения (4) при $c=a$, содержашемуся в $\overline{\psi S}$. Согласно условию теоремы $\eta=0$. Тогда $0 \in \overline{\psi S}$, что противоречит $S$-отделенности от нуля решения $\psi$. Значит, $\psi=0$ и мы находимся в условиях теоремы 1.

ЛЕмма 3. Пусть ч - ограниченное решение уравнения (3). Тогда уравнение (3) имеет ограниченное решение $\varphi_{0}$ такое, что $\overline{\varphi_{0}(P)} \subset \operatorname{co} \overline{\varphi(P)}$, и для произвольных решений $\psi_{1}$ и $\psi_{2} \in \overline{\varphi_{0} S}$ уравнения (3) выполняется соотношение

$$
\inf _{s \in S}\left\|\psi_{1}(s)-\psi_{2}(s)\right\|=0
$$

Если же для всех $(c, d) \in \overline{(a, b) S}$ выполнено равенство $\overline{(c, d) S}=\overline{(a, b) S}$, то соотношение (15) выполняется для произвольных решений $\psi_{1}$ и $\psi_{2} \in \overline{\varphi_{0} S}$ уравнения (5) при всех $(c, d) \in \overline{(a, b) S}$.

ДокАЗАТЕльСтво. Пусть $K=\operatorname{co} \overline{\varphi(P)}$ - вьпуклая оболочка множества $\overline{\varphi(P)}$. Тогда $K$ - компакт из $\mathbb{R}^{m}$. Через $X$ обозначим множество решений $\psi$ уравнения (3) таких, что $\overline{\psi(P)} \subset K$. Из леммы 2 следует, что множество $X$ компактно. Пусть

$$
r=\inf _{\psi \in X} \sup _{s \in S}\|\psi(s)\|
$$

Тогда

$$
r=\lim _{i \rightarrow \infty} \sup _{s \in S}\left\|\varphi_{i}(s)\right\|
$$

для некоторой последовательности $\left\{\varphi_{i}\right\} \subset X$. Считаем,

$$
\lim _{i \rightarrow \infty} \varphi_{i}=\varphi_{0} \in X
$$

Непосредственно доказьвается, что

$$
r=\sup _{s \in S}\left\|\varphi_{0}(s)\right\|
$$

Если $r=0$, то, очевидно, лемма доказана. Пусть $r \neq 0$ и $\psi_{1}$ и $\psi_{2} \in \overline{\varphi_{0} S}$ - решения уравнения (3). Тогда $\eta=\left(\psi_{1}+\psi_{2}\right) / 2$ - решение уравнения $(3)$. Поскольку $\overline{\varphi_{0}(P)} \subset K$ и $\psi_{1}=\lim _{i \rightarrow \infty} \varphi_{0 s_{i}}$ для некоторой направленности $\left\{s_{i}\right\} \subset S$, то для всех $t \in P$

$$
\psi_{1}(t)=\lim _{i \rightarrow \infty} \varphi_{0}\left(s_{i}+t\right) \in \overline{\varphi_{0}(P)} \subset K .
$$

Поэтому $\psi_{1}(t) \in K$ для всех $t \in P$. Аналогично, $\psi_{2}(t) \in K$ для всех $t \in P$. В таком случае $\eta(t)=\left(\psi_{1}(t)+\psi_{2}(t)\right) / 2 \in K$ и, следовательно, $\eta \in X$. Поэтому

$$
\sup _{s \in S}\|\eta(s)\| \geqslant r
$$


Поскольку для всех $s \in S$

$$
\left\|\psi_{1}(s)\right\|=\left\|\lim _{i \rightarrow \infty} \varphi_{0 s_{i}}(s)\right\| \leqslant \sup _{\tau \in S}\left\|\varphi_{0}(\tau)\right\|=r,
$$

то $\left\|\psi_{1}(s)\right\| \leqslant r$ для всех $s \in S$. Аналогично, $\left\|\psi_{2}(s)\right\| \leqslant r$ для всех $s \in S$. Поскольку

$$
\sup _{s \in S}\|\eta(s)\| \geqslant r
$$

то для любого $i \in \mathbb{N}$ существует $t_{i} \in S$ такое, что $\left\|\eta\left(t_{i}\right)\right\|>r-1 / i$. Поэтому

$$
2 r-\frac{2}{i}<2\left\|\eta\left(t_{i}\right)\right\|=\left\|\psi_{1}\left(t_{i}\right)+\psi_{2}\left(t_{i}\right)\right\| \leqslant\left\|\psi_{1}\left(t_{i}\right)\right\|+\left\|\psi_{2}\left(t_{i}\right)\right\| \leqslant 2 r<2 r+\frac{2}{i} ;
$$

следовательно, для всех $i \in \mathbb{N}$

$$
2 r-\frac{2}{i}<\left\|\psi_{1}\left(t_{i}\right)+\psi_{2}\left(t_{i}\right)\right\|<2 r+\frac{2}{i}
$$

и поэтому

$$
\lim _{i \rightarrow \infty}\left\|\psi_{1}\left(t_{i}\right)+\psi_{2}\left(t_{i}\right)\right\|=2 r .
$$

А так как $\left\|\psi_{1}\left(t_{i}\right)\right\| \leqslant r$ и $\left\|\psi_{2}\left(t_{i}\right)\right\| \leqslant r$, на основании теоремы о диагоналях параллелограмма вьполняется соотношение

$$
\lim _{i \rightarrow \infty}\left\|\psi_{1}\left(t_{i}\right)-\psi_{2}\left(t_{i}\right)\right\|=0
$$

Следовательно, верно (15) и первое утверждение леммы доказано.

Пусть для всех $(c, d) \in \overline{(a, b) S}$ вьполнено $\overline{(c, d) S}=\overline{(a, b) S}$, и пусть $(c, d) \in \overline{(a, b) S}$. Тогда $(c, d)=\lim _{n}\left(a_{s_{n}}, b_{s_{n}}\right)$ для некоторой направленности $\left\{s_{n}\right\}$ в $S$. Понятно, что $\varphi_{s_{n}}$ решение уравнения

$$
\frac{\delta y(x)}{\delta x(t)}=a_{s_{n}}(x, t) y(x)+b_{s_{n}}(x, t) .
$$

Согласно лемме 2 из направленности $\left\{\varphi_{0_{n}}\right\}$ можно выделить сходящуюся поднаправленность $\left\{\varphi_{0_{s_{n_{i}}}}\right\}$. Если $\psi=\lim _{i} \varphi_{0_{n_{i}}}$, то $\psi$ - решение уравнения (5), содержащееся в множестве $\overline{\varphi_{0} S}$. Предположим, что $\psi_{1}$ и $\psi_{2} \in \overline{\varphi_{0} S}$ - решения уравнения (5). Тогда $\eta=\left(\psi_{1}+\psi_{2}\right) / 2-$ решение уравнения (5) и для всех $t \in P$ вьполнено $\eta(t) \in K$. Поскольку $(a, b) \in \overline{(c, d) S}$, из леммы 2 следует, что существует решение $\delta$ уравнения (3), причем $\delta \in \overline{\eta S}$. Так как $\eta(t) \in K$ для всех $t \in P$, то из $\delta \in \overline{\eta S}$ следует, что $\delta(t) \in K$ для всех $t \in P$. Мы доказали, что $\delta \in X$, поэтому

$$
\sup _{s \in S}\|\delta(s)\| \geqslant r
$$

и, следовательно, для всех $i \in \mathbb{N}$ существует $t_{i} \in S$ такое, что $\left\|\delta\left(t_{i}\right)\right\|>r-1 / i$. Пусть

$$
\delta=\lim _{k \rightarrow \infty} \eta_{\tau_{k}}
$$


для $\left\{\tau_{k}\right\} \subset S$. Поскольку, очевидно, $\left\|\psi_{1}(s)\right\| \leqslant r$ и $\left\|\psi_{2}(s)\right\| \leqslant r$ для любого $s \in S$, то для всех $i \in \mathbb{N}$

$$
\begin{aligned}
r-\frac{1}{i} & <\left\|\delta\left(t_{i}\right)\right\|=\left\|\lim _{k} \eta_{\tau_{k}}\left(t_{i}\right)\right\|=\lim _{k}\left\|\eta\left(\tau_{k}+t_{i}\right)\right\|=\frac{1}{2} \lim _{k}\left\|\psi_{1}\left(\tau_{k}+t_{i}\right)+\psi_{2}\left(\tau_{k}+t_{i}\right)\right\| \\
& \leqslant \frac{1}{2} \lim _{k}\left\|\psi_{1}\left(\tau_{k}+t_{i}\right)\right\|+\frac{1}{2} \lim _{k}\left\|\psi_{2}\left(\tau_{k}+t_{i}\right)\right\| \\
& \leqslant \frac{1}{2} \sup _{t \in S}\left\|\psi_{1}(t)\right\|+\frac{1}{2} \sup _{t \in S}\left\|\psi_{2}(t)\right\| \leqslant r<r+\frac{1}{i} ;
\end{aligned}
$$

следовательно,

$$
2 r-\frac{2}{i}<\lim _{k}\left\|\psi_{1}\left(\tau_{k}+t_{i}\right)+\psi_{2}\left(\tau_{k}+t_{i}\right)\right\|<2 r+\frac{2}{i}
$$

и поэтому

$$
\lim _{i \rightarrow \infty} \lim _{k}\left\|\psi_{1}\left(\tau_{k}+t_{i}\right)+\psi_{2}\left(\tau_{k}+t_{i}\right)\right\|=2 r
$$

Тогда

$$
\lim _{i \rightarrow \infty}\left\|\psi_{1}\left(\tau_{k_{i}}+t_{i}\right)+\psi_{2}\left(\tau_{k_{i}}+t_{i}\right)\right\|=2 r
$$

для некоторой подпоследовательности $\left\{\tau_{k_{i}}\right\}$ направленности $\left\{\tau_{k}\right\}$. А так как

$$
\left\|\psi_{1}\left(\tau_{k_{i}}+t_{i}\right)\right\| \leqslant r \quad \text { и } \quad\left\|\psi_{2}\left(\tau_{k_{i}}+t_{i}\right)\right\| \leqslant r
$$

то, как и ранее, получим

$$
\lim _{i \rightarrow \infty}\left\|\psi_{1}\left(\tau_{k_{i}}+t_{i}\right)-\psi_{2}\left(\tau_{k_{i}}+t_{i}\right)\right\|=0
$$

следовательно,

$$
\inf _{s \in S}\left\|\psi_{1}(s)-\psi_{2}(s)\right\|=0
$$

и лемма доказана.

Теорема 3. Пусть ч - ограниченное решение уравнения (3). Тогда

1) если каждое ненулевое ограниченное решение уравнения (4) при с = $S$-отделено от нуля, то уравнение (3) имеет ограниченное $S$-согласованное с $(a, b)$ решение $\varphi_{0}$, причем $\overline{\varphi_{0}(P)} \subset \operatorname{co} \overline{\varphi(P)}$;

2) если отображение $(a, b)$ S-устойчиво по Лагранжу, для произвольного $(c, d) \in \overline{(a, b) S} \overline{(c, d) S}=\overline{(a, b) S}$ и для всех $c \in \overline{a S}$ каждое ненулевое ограниченное решение уравнения (4) $S$-отделено от нуля, то для всех $(c, d) \in$ $\overline{(a, b) S}$ уравнение $(5)$ имеет в мноэсестве $\overline{\varphi_{0} S}$ единственное ограниченное $S$-устойчивое по Лагранжу равномерно $S$-согласованное $c(c, d)$ решение. 
ДокАЗАТЕЛЬСтво. Пусть $\varphi_{0}$ - ограниченное решение уравнения (3) из леммы 3.

Докажем первое утверждение теоремы. Преж де всего докажем, что в множестве $\overline{\varphi_{0} S}$ нет решений уравнения (3), отличных от $\varphi_{0}$. Предположим, что $\psi \in \overline{\varphi_{0} S}$ - решение уравнения $(3)$ и $\psi \neq \varphi_{0}$. Тогда $\psi-\varphi_{0}$ - ненулевое ограниченное решение уравнения (4) при $c=a$ и, следовательно, согласно условию теоремы

$$
\inf _{s \in S}\left\|\psi(s)-\varphi_{0}(s)\right\|>0 .
$$

Это соотношение противоречит соотношению (15). Значит, $\psi=\varphi_{0}$.

Предположим, что $\varphi_{0}$ не $S$-согласовано с $(a, b)$. Тогда существует окружение $\alpha$ равномерной структуры пространства $C_{c}\left(P, \mathbb{R}^{m}\right)$ такое, что для произвольного окружения $\beta$ равномерной структуры пространства $C_{c}\left(P \times D, L_{b}\left(\mathbb{R}^{m}, \mathbb{R}^{m}\right) \times \mathbb{R}^{m}\right)$ существует элемент $s_{\beta} \in S$ такой, что

$$
\begin{gathered}
\left((a, b),\left(a_{s_{\beta}}, b_{s_{\beta}}\right)\right) \in \beta, \\
\left(\varphi_{0}, \varphi_{0_{s_{\beta}}}\right) \notin \alpha .
\end{gathered}
$$

Из соотношения (16) следует, что

$$
\lim _{\beta}\left(a_{s_{\beta}}, b_{s_{\beta}}\right)=(a, b) .
$$

В силу леммы замкнутости 2 из направленности $\varphi_{s_{\beta}}$ можно выделить поднаправленность $\varphi_{s_{\gamma}}$, сходящуюся к некоторому ограниченному решению $\psi$ уравнения $(3)$, содержащемуся в множестве $\overline{\varphi_{0} S}$. Согласно доказанному выше $\psi=\varphi_{0}$, поэтому

$$
\lim _{\gamma} \varphi_{0 s_{\gamma}}=\varphi_{0}
$$

Полученное соотношение противоречит (17). Противоречие говорит о том, что $\varphi_{0} S$-согласовано с $(a, b)$.

Докажем второе утверждение теоремы. Прежде всего заметим, что в силу леммы 3 и условий теоремы для каждой пары $(c, d)$ в множестве $\overline{\varphi_{0} S}$ содержится ровно по одному решению уравнения (5). Кроме этого, для всех $(c, d) \in \overline{(a, b) S}$ уравнение (5) удовлетворяет тем же условиям, что и уравнение (3). Поэтому для доказательства второго утверждения теоремы достаточно доказать, что $\varphi_{0}$ равномерно $S$-согласовано с $(a, b)$ и $S$-устойчиво по Лагранжу. Предположим, что $\varphi_{0}$ не равномерно $S$-согласовано с $(a, b)$. Тогда существует окружение $\alpha$ равномерной структуры пространства $C_{c}\left(P, \mathbb{R}^{m}\right)$ такое, что для произвольного окружения $\beta$ равномерной структуры пространства $C_{c}(P \times D$, $\left.L_{b}\left(\mathbb{R}^{m}, \mathbb{R}^{m}\right) \times \mathbb{R}^{m}\right)$ существуют элементы $s_{\beta}, t_{\beta} \in S$ такие, что

$$
\begin{gathered}
\left(\left(a_{t_{\beta}}, b_{t_{\beta}}\right),\left(a_{s_{\beta}}, b_{s_{\beta}}\right)\right) \in \beta, \\
\left(\varphi_{0_{t_{\beta}}}, \varphi_{0_{s_{\beta}}}\right) \notin \alpha .
\end{gathered}
$$

В силу компактности множества $\overline{(a, b) S}$ можем считать, что направленности $\left\{\left(a_{t_{\beta}}, b_{t_{\beta}}\right)\right\}$ и $\left\{\left(a_{s_{\beta}}, b_{s_{\beta}}\right)\right\}$ являются сходящимися. Пусть

$$
\lim _{\beta}\left(a_{t_{\beta}}, b_{t_{\beta}}\right)=\left(a_{1}, b_{1}\right) \quad \text { и } \quad \lim _{\beta}\left(a_{s_{\beta}}, b_{s_{\beta}}\right)=\left(a_{2}, b_{2}\right) .
$$


В таком случае из соотношения $(18)$ получаем, что $\left(a_{1}, b_{1}\right)=\left(a_{2}, b_{2}\right)=(c, d)$. В силу леммы замкнутости 2 можем считать, что

$$
\lim _{\beta} \varphi_{0} t_{\beta}=\psi_{1} \quad \text { и } \quad \lim _{\beta} \varphi_{0} s_{\beta}=\psi_{2},
$$

при этом $\psi_{1}$ и $\psi_{2}$ - решения уравнения (5). Но тогда $\psi_{1}=\psi_{2}$, что противоречит (19). Значит, $\varphi_{0}$ равномерно $S$-согласовано с $(a, b)$. Решение $\varphi_{0} S$-устойчиво по Лагранжу в силу леммы 3.

\section{СПИСОК ЦИТИРОВАННОЙ ЛИТЕРАТУРЫ}

[1] Гайшун И. В. Вполне разрешимые многомерные дифференциальные уравнения. Минск: Наука и техника, 1983.

[2] Шербаков Б.А. Многомерные динамические системы // Дифференц. уравнения. 1994. T. 30. № 5. C. 739-747.

[3] Герко А. И. Согласованные решения линейных многомерных дифференциальных уравнений // Изв. АН Респ. Молдова. Сер. матем. 1994. №1. С. 42-47.

[4] Герко А.И. Согласованные решения квазилинейных многомерных дифференциальных уравнений // Изв. АН Респ. Молдова. Сер. матем. 1995. № 1. С. 3-11.

[5] Хынку В.И.Приводимость линейных вполне интегрируемых систем с квазипериодическими коэффициентами // Изв. АН Респ. Молдова. Сер. матем. 1996. №1. С. 24-30.

[6] Glavan V. A., Hâncu V. I. Partial recurrent solutions of completely integrable systems // Изв. АН Респ. Молдова. Сер. матем. 1998. № 1. С. 103-112.

[7] Герко А. И. Асимптотически рекуррентные решения уравнений в $\beta$-производных // Матем. заметки. 2000. Т. 67. №6. С. 837-851.

[8] Задорожний В.Г. Вполне интегрируемые уравнения в вариационных производных // Дифференц. уравнения. 1975. Т. 11. №11. С. 2027-2039.

[9] Гайшун И.В. Линейные уравнения в полных производных. Минск: Наука и техника, 1989.

[10] Шербаков Б. А. Устойчивость по Пуассону движений динамических систем и решений дифференциальных уравнений. Кишинев: Штиинца, 1985.

[11] Левитан Б. М., ЖКиков В. В. Почти-периодические функции и дифференциальные уравнения. М.: Изд-во МГУ, 1978.

[12] Бронштейн И.У. Расширения минимальных групп преобразований. Кишинев: Штиинца, 1975.

[13] Gerko A. I. Poisson stability of mappings with respect to a semigroup // Изв. АН Респ. Молдова. Сер. матем. 1998. №1. С. 95-102.

Государственный университет Молдовы, Кишинев

Поступило

E-mail : gerko@usm.md

02.04 .2002 